\title{
Assessment of Anxiety and Effect of Preemptive Analgesia on Patients Administered Ambulatory Anesthesia in Endoscopy Unit
}

\author{
(D) Ceyda Ozhan Caparlar, (1) Savaş Altınsoy, () Julide Ergil
}

Department of Anesthesiology and Reanimation, University of Health Sciences Turkey, Diskapi Yildirim Beyazit Training and Research Hospital, Ankara, Turkey

\begin{abstract}
Introduction: Endoscopy and colonoscopy are gold standards for the diagnosis and treatment of gastrointestinal diseases. The success of ambulatory gastrointestinal procedures rely on examination, the cooperation of the patient, drug selection, experience of anesthesiologists with endoscopists. This study aims to assess preoperative anxiety arising from endoscopy and colonoscopy applied successively in endoscopy units, to take precautions accordingly and to compare dexketoprofen and paracetamol for preemptive analgesia.

Methods: In this study, 150 patients (ASA I-II,18-60 years) who were scheduled to go under endoscopy and colonoscopy were included. Demographic data (gender, age, BMI), American Society of Anesthesiologists(ASA) scores and education background were recorded. State-Trait Anxiety Inventory I-II(STAI I-II) testes were applied to the patients.

Results: Patients were informed by the Numerical Rating Scale (NRS) in the endoscopy unit. Pregabalin was administered one hour before the procedure as per established procedures in all groups. Patients were divided into three groups, paracetamol in group $P(n=20)$, dexketoprofen in group D $(n=20)$, and saline in Group C $(n=20)$ were administered. At the end of the procedure, patients above 2, according to the Ramsay Sedation Scale (RSS), were taken to the recovery room. Their pain levels were measured at minutes 5, 30,60, and 120 following procedure using NRS. The times to reach above 9 of the modified Aldrete recovery score (MARS) were recorded. There was no difference concerning demographics, ASA, education, duration of anesthesia, endoscopist's and patient's satisfaction, duration of the procedure, and NRS levels. The time to reach MARS 9 was shorter in Group D than Group P, and C. Total propofol consumption throughout sedation was less in Group D compared to Group P and C.

Discussion and Conclusion: It is necessary to recognize the anxiety of the patients in the gastrointestinal procedure and to take precautions. By applying preemptive analgesia, the total anesthetic dose can be reduced, and discharge time can be shortened. Keywords: Ambulatory anesthesia; preemptive anesthesia; pregabaline.
\end{abstract}

$\mathrm{E}^{\mathrm{n}}$ ndoscopy and colonoscopy are the gold standards for the diagnosis and treatment of gastrointestinal diseases. Sedation and analgesia methods applied during ambulatory gastrointestinal procedures increase patient

comfort appreciably. In ambulatory anesthesia, which is applied during minimally invasive procedures, patients recover quickly and are discharged from the hospital and go back to their healthy life quickly ${ }^{[1]}$. For successful and

Correspondence (Iletişim): Ceyda Ozhan Caparlar, M.D. Saglik Bilimleri Universitesi Diskapi Yildirim Beyazit Egitim Arastirma Hastanesi, Anesteziyoloji ve Reanimasyon Klinigi, Ankara, Turkey Phone (Telefon): +90 5052919489 E-mail (E-posta): mdceydacaparlar@yahoo.com Submitted Date (Başvuru Tarihi): 16.06.2019 Accepted Date (Kabul Tarihi): 05.12.2019 Copyright 2020 Haydarpaşa Numune Medical Journal OPEN ACCESS This is an open access article under the CC BY-NC license (http://creativecommons.org/licenses/by-nc/4.0/). 
safe ambulatory anesthetic procedures, a variety of factors, such as patient selection, anesthesiologist's experience, anesthesia or analgesia method, and patient's post-treatment satisfaction, need to be considered. It can be possible to eliminate the patient's preoperative anxiety, reduce their anesthesia-related awareness, establish short-term amnesia, and prevent and mitigate complications that may occur during and after the procedure using the appropriate anesthesia and analgesia techniques.

In ambulatory gastrointestinal procedures requiring prolonged sedation, it must be made sure that an appropriate anesthetic depth is reached to prevent sudden movements while maintaining respiratory for patient safety.

In sedation and/or analgesia procedures, it is necessarily expected to keep patient safety at the forefront without creating a risk for the patient and to bring forward the time of discharge with a safe and fast recovery. For this purpose, propofol, opioids, benzodiazepines, analgesics, and antipsychotics can be used separately or used in combination $^{[2,3]}$. Here is literature evidence that multimodal analgesia is effective in ambulatory gastrointestinal procedures ${ }^{[3]}$. Pregabalin is a gamma-aminobutyric acid (GABA) analog influential on anxiety ${ }^{[4,5]}$. Pregabalin has been shown to replace the $[3 \mathrm{H}]$-gabapentin by binding to voltage-sensitive calcium channel subunits in the central nervous system (to $a 2-\delta$ protein). Pregabalin reduces the release of numerous neurotransmitters, including glutamate, noradrenaline, and substance $\mathrm{P}^{[6]}$.

Preemptive analgesia refers to prevention or reducing of pain by analgesics administered before painful stimulants. The goal is to prevent the formation of any pain memory in the nervous system and reduce the need for analgesics. Preemptive analgesia is not only a timing strategy but also about administering the drugs at the appropriate time, dose, manner, and duration. It helps prevent hypersensitization, which may develop in the patient and thus mitigate postoperative pain sense. Preemptive analgesia decreases preoperative stress, duration of hospitalization, costs, and postoperative morbidity ${ }^{[7]}$. Paracetamol is an analgesic and antipyretic nonsteroidal anti-inflammatory drug that inhibits cyclooxygenase selectively ${ }^{[8]}$. Intermittent paracetamol administration reduces the use of opioids consumed by a patient-controlled analgesia method. The analgesic activity depends on the blood concentration of the drug. Dexketoprofen is a water-soluble salt of the active rotated enantiomer of ketoprofen. It is in the aryl-propionic acid group. This type of ketoprofen is not an anti-inflammatory ${ }^{[9]}$. The analgesic effect starts quite fast. The lack of serious adverse effects, such as respiratory depression, has also led to the preference of the drug in ambulatory anesthesia. Dexketoprofen maximum plasma concentration is between 0.25 and 0.75 hours $^{[9]}$. Dexketoprofen has no adverse effects, such as bleeding time prolongation or platelet aggregation ${ }^{[10]}$.

This study aims to assess preoperative anxiety levels of the patients due to following endoscopy and colonoscopy procedures and also comparing dexketoprofen and paracetamol as pre-emptive analgesic agents.

\section{Materials and Methods}

This study was started after consent was obtained from the Ethics Committee of Dıskapı Yıldırım Beyazıt Training and Research Hospital of the Health Sciences University (25.09.2017/41.04), and informed consent certificate was obtained from the patients.

The study population consisted of 150 patients of ASA I-II (aged 18 to 60) who were about to go endoscopy and colonoscopy consecutively sedation-analgesia method in the General Surgery Clinic of Dıskapı Yıldırım Beyazıt Training and Research Hospital. This study was planned single centered, prospective, randomized, single-blind, and placebo-controlled.

The patients were excluded from this study if they from hypertension, cardiovascular disease, sleep apnea syndrome, active peptide ulcer, bleeding disorder, kidney and liver failure, history of paracetamol and dexketoprofen allergy, psychiatric disease, have a history of psychiatric medication, and if they receive chronic analgesia treatment. For all the patients admitted to the anesthesia clinic, specific data were recorded, such as age, sex, BMI (body mass index), ASA (American Society of Anesthesiologists) class, and level of education.

At the same time, the patients were given the 'State-Trait Anxiety Inventory I and II (STAI I-II) to measure their anxiety level (Tables 1, 2). The State-Trait Anxiety Inventory (STAI) was developed by Spielberger et al. ${ }^{[11]}$ in 1970 . In that test, high scores show higher levels of anxiety, but low scores indicate lower levels of anxiety. It is a four-point scale ranging from "Never" to "Completely." There are two types of expressions in the State-Trait Anxiety Inventory. In the scale, straight expressions refer to negative feelings, while reversed expressions to positive feelings. The items numbered $1,2,5,8,10,11,15,16,19$, and 20 are reversed items in the scale. The straight and reversed items have different total weights. The total weight score of the reverse items is subtracted from the total weight score of the straight items. 
Table 1. Demographic data

\begin{tabular}{|c|c|c|c|c|}
\hline & Group P $(n=50)$ & Group D $(n=50)$ & Group C $(n=50)$ & $\mathbf{p}$ \\
\hline \multicolumn{5}{|l|}{$\operatorname{Sex}(n, \%)$} \\
\hline Female & $26(52)$ & $36(72)$ & $26(52)$ & 0.199 \\
\hline Male & $24(48)$ & $14(28)$ & $24(48)$ & 0.321 \\
\hline Age (years, mean $\pm S D$ ) & $38.56 \pm 12.58$ & $36.28 \pm 13.72$ & $38.76 \pm 15.53$ & 0.54 \\
\hline $\mathrm{BMI}\left(\mathrm{m}^{2} / \mathrm{kg}\right.$, mean $\left.\pm \mathrm{SD}\right)$ & $26.42 \pm 4.45$ & $27.91 \pm 4.19$ & $27.89 \pm 4.44$ & 0.141 \\
\hline \multicolumn{5}{|l|}{ ASA $(n, \%)$} \\
\hline I & $21(42)$ & $19(38)$ & $21(42)$ & 0.951 \\
\hline ॥ & $29(58)$ & $31(62)$ & $29(58)$ & 0.951 \\
\hline \multicolumn{5}{|l|}{ Education (n, \%) } \\
\hline Elementary & $15(30)$ & $14(28)$ & $15(30)$ & 0.978 \\
\hline Secondary & $15(30)$ & $16(32)$ & $15(30)$ & 0.978 \\
\hline High School & $9(18)$ & $10(20)$ & $9(18)$ & 0.965 \\
\hline University & $11(22)$ & $10(20)$ & $11(22)$ & 0.969 \\
\hline Duration of anaesthesia (min, mean \pm SD) & $47.06 \pm 10.86$ & $44.52 \pm 8.7$ & $46.1 \pm 10.71$ & 0.530 \\
\hline Duration of procedure (min, mean \pm SD) & $37.14 \pm 10.99$ & $37.76 \pm 8.72$ & $38.17 \pm 10.02$ & 0.683 \\
\hline Period before modified Aldrete recovery score $>9(\mathrm{~min}$, mean $\pm \mathrm{SD})$ & $11.94 \pm 1.51$ & $10.78 \pm 1.28^{*}$ & $11.92 \pm 1.48$ & $<0.001$ \\
\hline Patient satisfaction (median, min-max) & $7(5-7)$ & $6(4-7)^{*}$ & $7(5-7)$ & 0.06 \\
\hline Endoscopist satisfaction (median, min-max) & $6(5-7)$ & $6(5-7)$ & $7(5-7)$ & 0.969 \\
\hline
\end{tabular}

*Group D has a significant difference in bonferroni correction compared to others.

Table 2. STAI scores

\begin{tabular}{|c|c|c|c|c|}
\hline & Group P $(n=50)$ & Group D $(n=50)$ & Group $C(n=50)$ & $\mathbf{p}$ \\
\hline STAI-2 (mean \pm SD) & $42.02 \pm 4.65$ & $41.8 \pm 6.23$ & $40.06 \pm 5.53$ & 0.069 \\
\hline Pre-operative STAI-1 (mean \pm SD) & $50.96 \pm 6.23$ & $54.58 \pm 10.98$ & $53.86 \pm 9.66$ & 0.418 \\
\hline Post-operative STAI-I (mean \pm SD) & $36.9 \pm 11.89$ & $40.8 \pm 12.34$ & $40.66 \pm 14.35$ & 0.228 \\
\hline \multicolumn{5}{|l|}{ NRS scores (median, min-max) } \\
\hline to & $3(1-5)$ & $2(1-4)$ & $3(1-5)$ & 0.05 \\
\hline t2 & $3(1-5)$ & $3(1-5)$ & $3(1-5)$ & 0.483 \\
\hline t3 & $1(0-3)$ & $1(0-3)$ & $1(0-2)$ & 0.288 \\
\hline
\end{tabular}

A predetermined and constant value is added to this figure. This constant value is $\mathbf{5 0}$ for the State-Trait Anxiety Inventory. This value represents the individual's anxiety score. The State-Trait Anxiety Inventory (STAI) is a susceptible tool for evaluating sudden changes in emotional reactions. The scores range between 20 (low anxiety) and 80 (high anxiety). The form was read to the patients, and the responses were noted by marking the appropriate expressions corresponding to the situational anxiety level by the researcher. If more than three questions were not answered in the STAI form, the completed form was considered invalid and not scored. After answering the questions, the STAl score was calculated and recorded. Anxiety classification was performed between lower (STAI <20) and a higher level of anx- iety (STAI>80). Only those with the STAI-1 levels above 45 were included in this study.

When the patients were in the endoscopy unit, they were instructed on the use of the NRS to assess pain ( $0=$ no pain; $10=$ worst pain imaginable) and then Pregabalin (Lyrica $150 \mathrm{mg}$ capsule) was administered to all of them one hour before the procedure as a part of the regular protocol. The participants were put in Group P, Group D, and Group C, which were determined randomly with the computer. Paracetamol (1000 mg) (Perfalgan, Bristol-Myers Squibb, United States); $1 \mathrm{~g} / 150 \mathrm{ml}$ Saline/10 min infusion in group P, Dexketoprofen $(50 \mathrm{mg}$ ) (Arveles, IE Ulagay-Menarini, Turkey); $50 \mathrm{mg} / 150 \mathrm{ml}$ standard saline/10 min infusion in group $D$ and 150 cc saline was administered in group $C$. 
Standard anesthesia techniques were used for all patients in the endoscopy unit. Heart rate (HR), mean arterial pressure (MAP), oxygen saturation $\left(\mathrm{SpO}_{2}\right)$, and end-tidal carbon dioxide $\left(\mathrm{ETCO}_{2}\right)$ were continuously monitored.

Before the endoscopy, the patients were placed in the prone position facing the endoscopist with a mouth opener big enough to allow endoscope pass. Then $4 \mathrm{~L} /$ min oxygen inlet was started with a nasal cannula. During the anesthesia induction, $1 \mathrm{mg} / \mathrm{kg}$ propofol and $1 \mathrm{mcg} / \mathrm{kg}$ fentanyl were given. For the maintenance of anesthesia, propofol infusion was administered at a dose of $(1-3 \mathrm{mg} /$ $\mathrm{kg}$ ) titrated according to the BIS levels between 40 and 60 .

Post-procedure pain (patients with an NRS score of 4 or more) was managed with intravenous tramadol $(50 \mathrm{mg})$ as rescue analgesia ${ }^{[12]}$. Colonoscopy was continued in the same position upon completion of the endoscopy. The hemodynamic data were monitored at 10-minute intervals throughout the intra-operative period. Hypotension was defined as a decrease of at least $20 \%$ in the MAP from baseline values and was treated with intravenous ephedrine 5 mg. Bradycardia was defined as HR of fewer than 45 beats per minute and was treated with intravenous atropine $0.5 \mathrm{mg}$. Additional analgesic requirements and complications were recorded. When the successive endoscopy and colonoscopy procedures ended, the patients were assessed with the Ramsey Sedation Scale (RSS) by the anesthesiologist. When the RSS score was below 2, the patients were sent to the recovery room. NRS was measured at minutes 5 (t0), $30(\mathrm{t} 1), 60(\mathrm{t} 2)$, and $120(\mathrm{t} 3)$, respectively, after the procedure.

When four hours passed after the procedure, the STAI-I questionary was conducted to all patients to determine the anxiety levels, and their responses were recorded. Both patients and endoscopist's satisfaction were assessed before discharge using a 7 point ( $1=$ not satisfied, $7=$ really satisfied) Likert scale ${ }^{[13]}$ and recorded. The time of reaching 9 in the modified Aldrete recovery score was also recorded so that the patients with a score equal to or above 9 could be released from the recovery unit.

\section{Statistical Analysis}

According to our preliminary study data, the rate of patients who needed additional analgesia in the control group was $45 \%$. If there is a decrease of $60 \%$ in the number of patients in need of new analgesics, 49 patients must be taken for each group $(a=0.05, b=0.20)$. Therefore, 56 patients were included in each group not to face a problem because of the probable high number of exclusions.
The statistical analysis was performed using the Statistical Program for Social Sciences (SPSS, version 22.0, Chicago, IL, USA). The numerical values were given as mean \pm standard deviation. Normality was checked with the Shapiro-Wilk test. In cases with normal distribution for continuous variables at univariate intervals, the Student t-test was used, while it was replaced with Mann-Whitey $U$ and Kruskal when distribution was not normal. As for the categorical variables, they were treated with Fisher exact test or chisquare test. The results were regarded as significant at the level of $p<0.05$.

\section{Results}

In this study, 168 patients were interviewed. Eight patients refused to participate in this study, and 10 patients had the only endoscopy without colonoscopy. Overall, 150 patients who met the inclusion criteria were included in this study.

Demographic data (gender, age, and BMI), ASA, educational status, the time of reaching 9 in the MARS, duration of anesthesia, duration of the procedure, endoscopist's satisfaction and patient's satisfaction are demonstrated in Table 1. As a result, there were no statistically significant differences between the groups for demographic data. There were also no significant differences between-group in ASA status, duration of anesthesia, and procedure. There were no differences between groups in endoscopists' satisfaction. However, the patient's satisfaction score was lower in group $D$ than the other groups. The time of MARS $>9$ was shorter in group $D$ than the other groups in table 1 $(\mathrm{p}<0.05)$.

STAI and NRS score levels were demonstrated was similar between the groups in Table 2. Preoperative STAI- I and STAI- II scores were similar between the groups. Although postoperative STAI-I values were lower compared to preoperative values, there was no significant difference between the groups. Concerning the NRS score, there was no significant difference between the groups.

Total propofol consumption and additional rescue analgesic count are demonstrated in Table 3. As a result, total propofol consumption decreased in Group D compared to Group P and Group C $(p<0.05)$. For rescue analgesic count, no difference was found between Group P and Group D; however, the need in these groups was lower in comparison to Group C $(p<0.05)$.

No side effects (nausea, vomiting) were observed in this study. There was no statistically significant difference between the groups in hemodynamic variables. 
Table 3. Need for propofol and additional analgesics by groups

\begin{tabular}{lccr}
\hline & Group P (n=50) & Group D (n=50) & Group C (n=50) \\
\hline Propofol consumption (mg, mean \pm SD) & $133.9 \pm 13.89$ & $131.3 \pm 18.25$ & $140.74 \pm 15.12$ \\
Need for additional analgesics (n, \%) & & & 0.007 \\
Yes & $10(20)^{*}$ & $6(12)^{*}$ & $19(38)$ \\
No & $40(80)$ & $44(88)$ & $31(62)$ \\
\hline
\end{tabular}

*Group D and Group P were significantly lower than Group C. No difference between Group P and Group D, Group D was significantly lower than Group P and Group C $(p<0.05)$.

\section{Discussion}

Ambulatory gastrointestinal procedures, effective sedation, analgesia methods must be employed to reduce patients' perceived pain and anxiety during and after the procedure. In ambulatory anesthesia administration, the shortage of sedation increases the risk of consciousness during anesthesia ${ }^{[14]}$. Patient safety must be brought to the forefront while ensuring the adequate anesthetic depth effective and appropriate anesthesia methods should be applied to decrease the consciousness during anesthesia.

In general, preoperative anxiety augments postoperative pain and delays recovery and discharge from the hospital. Regardless of the magnitude of the procedure, patients experience different levels of anxiety and fear at the preoperative stage. In a previous study, anxiety was reported before the procedure by $60-80 \%$ adult patients and $50-70 \%$ of young patients ${ }^{[15]}$. Preoperative anxiety among patients varies depending on the patient's purpose for visiting the hospital, gender, anesthesia management, and manner of surgery ${ }^{[16]}$. In the present study, clinical examination was performed on patients before outpatient endoscopy and colonoscopy consecutively. It was found out that STAI-II scores, which show constant anxiety, were similar in all the participating groups; still, none of the patients reported higher levels of anxiety. Unlike the previous, STAI-I scores, which refer to state anxiety levels, were found to be high in all groups before the procedure. We think that the present study is significant because it highlights the anxiety of patients before the medical procedures even though they do not show a high level of anxiety in their everyday life. This finding may arise from many factors, such as the likelihood of diagnosing the reason of symptoms or findings, going on a special diet for preparing the bowels, laxative administration, the concern about probable pain during and after the procedure, and patient's discomfort with the procedural position because of their ethical values. Before the procedure to contribute to both anxiolytic and analgesics, pregabalin $(150 \mathrm{mg}$ ) was implemented as a single dose.
Pregabalin is classically used in the treatment of epilepsy, partial convulsion, anxiety, and neuropathic pain, and recently in acute pain treatment. Furthermore, studies suggest that pregabalin can also be used in the treatment of postoperative pain ${ }^{[4]}$. In a meta-analysis study, Han et al. ${ }^{[17]}$ performed a comparison of the drugs used for acute pain treatment and concluded that pregabalin, also known as an adjuvant, increases analgesic outcome.

Bach et al. ${ }^{[5]}$ compared pregabalin with valproic acid for anxiety, and they observed similar anxiolytic properties in both analgesic substances. Hetta et al., ${ }^{[13]}$ in a study on patients going under mastectomy, compared the effects of differing doses of preoperative pregabalin on pain intensity and overall opioid consumption. They noted that pregabalin decreases opioid consumption while having an analgesic effect. In another study, Fujita et al. ${ }^{[14]}$ administered diazepam $5 \mathrm{mg}$ (placebo), pregabalin $75 \mathrm{mg}$ or 150 $\mathrm{mg}$ and they found lower levels of early postoperative visual analog scale (VAS) scores and morphine consumption in the $150 \mathrm{mg}$ pregabalin group than diazepam group. Although there is an abundance of research on monitoring patients' preoperative anxiety with the aid of STAI-I, studies are quite rare, which applies STAI-II to in the same patients. The literature offers a limited number of studies that distinguish between permanent anxiety and state anxiety during polyclinic evaluation and administer preoperative anxiolytic pregabalin. Our study seems to be one of the unique examples in this regard. Preoperative anxiety both increases patient awareness of anesthesia and also jeopardizes intra-operative and postoperative patient safety. However, we could manage to decrease preoperative anxiety using anxiolytic pregabalin through our procedures in this study.

The use of a multimodal approach in the management of preemptive analgesia has reduced anesthetic drug consumption, which has many side effects, but also led to more successful results in postoperative analgesia method. Solmaz and Kavalak ${ }^{[18]}$ found out that combined use of parac- 
etamol and tramadol functions better than the use of the drugs alone in ambulatory anesthesia. Tinsbloom et al. ${ }^{[3]}$ pointed out that the use of opioids only for the analgesic purpose in ambulatory treatment could increase both duration of recovery and opioid addiction, but the addition of gabapentin decreased opioid use and thus shortened the recovery duration further. Rana et al. ${ }^{[19]}$ noted that the use of nonsteroidal parecoxib against postoperative hysterectomy pain could reduce opioid consumption and led to an opioid-sparing effect. In our study, paracetamol and dexketoprofen were used for the preemptive analgesic outcome. Both analgesics were preferred in this study since they were immune from severe side effects like respiratory depression, the onset of the effect is soon, and the intravenous form of both drugs are readily available in Turkey. Paracetamol is preferred due to the ease of administration of form IV, less gastrointestinal side effects, and the similarity of the analgesic efficacy with other NSAI drugs. Kharouba et al., ${ }^{[20]}$ in their study, observed superiority of paracetamol and ibuprofen over placebo in relieving post-procedural pain in pediatric dental procedures. Also, Munro et al. ${ }^{[21]}$ compared paracetamol, ketamine, and GABA analogs and found out that paracetamol is also superior to multimodal analgesia. Ridderikhof et al. ${ }^{[22]}$ conducted a study on pain treatment caused by acute minor muscle-skeleton extremity traumas and concluded that the use of paracetamol shows similar benefits to diclofenac or combination of acetaminophen and diclofenac, eliminating the pain both in action and rest.

Sedation means different levels of confusion realized through medication. It is performed in three levels as mild, moderate, and deep ${ }^{[1]}$. It is recommended to monitor the level of sedation to be able to prevent unwanted effects (awareness during anesthesia, hypertension). For this purpose, the BIS VISTA monitor was used periodically, and the sedative drugs were titrated according to the BIS levels between 40 and 60 . After procedures were accompanied by sedation, the patient's deep sedation and analgesia should be maintained without compromising the airway reflexes. Mild and moderate levels of sedation can be sufficient for either endoscopy or colonoscopy procedures.

In this study, several anesthetic drugs were used to ensure sedation during endoscopy and colonoscopy. In different studies, Triantafilidis et al. ${ }^{[23]}$ found that deep sedation was noted in long-duration gastroenterological procedures. During deep sedation on ambulatory patients, cardiovascular functions can be maintained and considered the response to painful and repetitive stimuli may be obtained; nevertheless, the procedure to the airway may be neces- sary due to the probability of spontaneous respiration insufficiency ${ }^{[1]}$. In the current study, lower amounts of propofol consumption were attained, and recovery duration was shortened since the clinical evaluation contained giving of tests to increase anxiety awareness and preemptive analgesia. So far, many analgesic methods have been tested in ambulatory endoscopy and colonoscopy procedures. Although many studies have shown the use of propofol, a hypnotic agent is superior to other hypnotics, especially concerning the recovery, it has been reported that its pure use has not provided an adequate anesthetic depth ${ }^{[24]}$. In endosonographic ultrasonography (EUS) and upper gastrointestinal procedures, sedation has been achieved through hypnotic agents such as midazolam and opioids, but the recovery period has been prolonged a lot ${ }^{[25]}$.

In this study, paracetamol and dexketoprofen were applied as preemptive analgesics. Propofol was administered lower in group D than group P and group C. Moreover, considering the MARS, the dexketoprofen group could reach the score of and above 9 sooner than the other groups. On this account, we think the use of dexketoprofen leads to a decrease in the use of anesthetic drug consumption as well as in the recovery period. In the case of ambulatory procedures, a difference can be determined in hemodynamic values depending on the degree of sedation ${ }^{[26]}$. Besides, despite higher satisfaction among patients and endoscopists in Group D compared to Group P and Group C, the difference was not significant. No complications were encountered during the intraoperative and postoperative follow-up period in both groups. No complications were encountered duration in the endoscopy and recovery unit. The limitation of this study is that we did not assess the history of the previous endoscopy and colonoscopy procedures and the number of the previous endoscopy and colonoscopy procedures. Also, anxiety and pain were seen in many patients with different severities in operation.

\section{Conclusion}

In endoscopy units, gastrointestinal cases performed under ambulatory anesthesia require higher levels of attention. We suggest considering patients' anxiety levels during the preoperative assessment of outpatient anesthetic cases. In general, multimodal anesthetic and analgesic drugs have been used as a part of ambulatory anesthetic procedures performed in endoscopy units. Besides reaching sufficient anesthetic depth, it is vital to ensure the patient's inaction for the success of the operation.

In particular cases of ambulatory gastrointestinal proce- 
dures, it is possible to diminish the number of anesthetic drug doses and period before discharge if attention is paid to the patients' anxiety, diagnosing, taking necessary precautions, and applying preemptive analgesia. As a result, it is of great importance that the anesthesiologist and endoscopist undertake preoperative assessment carefully and collaborate throughout the procedure.

Ethics Committee Approval: This study was started after consent was obtained from the Ethics Committee of Dıskapı Yıldırım Beyazit Training and Research Hospital of the Health Sciences University (25.09.2017/41.04), and informed consent certificate was obtained from the patients.

Peer-review: Externally peer-reviewed.

Authorship Contributions: Concept: C.O.Ç., S.A.; Design: C.O.Ç., S.A.; Data Collection or Processing: S.A.; Analysis or Interpretation: J.E.; Literature Search: S.A.; Writing: C.O.Ç.

Conflict of Interest: None declared.

Financial Disclosure: The authors declared that this study received no financial support.

\section{References}

1. Obara K, Haruma K, Irisawa A, Kaise M, Gotoda T, Sugiyama $M$, et al. Guidelines for sedation in gastroenterological endoscopy. Dig Endosc 2015;27:435-49. [CrossRef]

2. Zhang $\mathrm{R}, \mathrm{Lu} \mathrm{Q}, \mathrm{Wu}$ Y. The Comparison of Midazolam and Propofol in Gastrointestinal Endoscopy: A Systematic Review and Meta-analysis. Surg Laparosc Endosc Percutan Tech 2018;28:153-8. [CrossRef]

3. Tinsbloom B, Muckler VC, Stoeckel WT, Whitehurst RL, Morgan B. Evaluating the Implementation of a Preemptive, Multimodal Analgesia Protocol in a Plastic Surgery Office. Plast Surg Nurs 2017;37:137-43. [CrossRef]

4. Clarke H, Pagé GM, McCartney CJ, Huang A, Stratford P, Andrion J, et al. Pregabalin reduces postoperative opioid consumption and pain for 1 week after hospital discharge, but does not affect function at 6 weeks or 3 months after total hip arthroplasty. Br J Anaesth 2015;115:903-11. [CrossRef]

5. Bach DR, Korn CW, Vunder J, Bantel A. Effect of valproate and pregabalin on human anxiety-like behaviour in a randomised controlled trial. Transl Psychiatry 2018;8:157. [CrossRef]

6. Markman J, Resnick M, Greenberg S, Katz N, Yang R, Scavone $J$, et al. Efficacy of pregabalin in post-traumatic peripheral neuropathic pain: a randomized, double-blind, placebo-controlled phase 3 trial. J Neurol 2018;265:2815-24. [CrossRef]

7. Wang $N$, Wang Y, Pang $L$, Wang J. Effect of preemptive analgesia with intravenous oxycodone in the patients undergoing laparoscopic resection of ovarian tumor. Pak J Med Sci 2015;31:300-3.

8. Hinz B, Cheremina O, Brune K. Acetaminophen (paraceta$\mathrm{mol}$ ) is a selective cyclooxygenase-2 inhibitor in man. FASEB J 2008;22:383-90. [CrossRef]

9. Rodríguez MJ, Arbós RM, Amaro SR. Dexketoprofen trometa- mol: clinical evidence supporting its role as a painkiller. Expert Rev Neurother 2008;8:1625-40. [CrossRef]

10. Von Plato H, Mattila K, Poikola S, Löyttyniemi E, Hamunen K, Kontinen $\mathrm{V}$. Risk-based targeting of adjuvant pregabalin treatment in laparoscopic cholecystectomy: A randomized, controlled trial. Scand J Pain 2019. [CrossRef]

11. Jeppesen E, Pedersen CM, Larsen KR, Walsted ES, Rehl A, Ehrenreich J, et al. Listening to music prior to bronchoscopy reduces anxiety - a randomised controlled trial. Eur Clin Respir J 2019;6:1583517. [CrossRef]

12. Kim MK, Moon HY, Ryu CG, Kang H, Lee HJ, Shin HY. The analgesic efficacy of the continuous adductor canal block compared to continuous intravenous fentanyl infusion with a single-shot adductor canal block in total knee arthroplasty: A randomized controlled trial. Korean J Pain 2019;32:30-8.

13. Yeung AWK, Wong NSM. The Historical Roots of Visual Analog Scale in Psychology as Revealed by Reference Publication Year Spectroscopy. Front Hum Neurosci 2019;13:86.

14. Pandit JJ, Andrade J, Bogod DG, Hitchman JM, Jonker WR, Lucas N, et al; Royal College of Anaesthetists; Association of Anaesthetists of Great Britain and Ireland. 5th National Audit Project (NAP5) on accidental awareness during general anaesthesia: summary of main findings and risk factors. $\mathrm{Br} J$ Anaesth 2014;113:549-59. [CrossRef]

15. Lin CJ, Liu HP, Wang PY, Yu MH, Lu MC, Hsieh LY, et al. The Effectiveness of Preoperative Preparation for Improving Perioperative Outcomes in Children and Caregivers. Behav Modif 2019;43:311-29. [CrossRef]

16. Ihde-Scholl T, Amsler F, Harms C, Scheidegger D, Kindler $\mathrm{CH}$. The Visual Analog Scale Allows Effective Measurement of Preoperative Anxiety and Detection of Patients' Anesthetic Concerns. Anesth Analg 2004;90:706-12. [CrossRef]

17. Han C, Kuang MJ, Ma JX, Ma XL. Is pregabalin effective and safe in total knee arthroplasty? A PRISMA-compliant metaanalysis of randomized-controlled trials. Medicine (Baltimore) 2017;96:e6947. [CrossRef]

18. SolmazFA, KovalakE. Comparison of tramadol/acetaminophen fixed-dose combination, tramadol, and acetaminophen in patients undergoing ambulatory arthroscopic meniscectomy. Acta Orthop Traumatol Turc 2018;52:222-5. [CrossRef]

19. Rana MV, Desai R, Tran L, Davis D. Perioperative Pain Control in the Ambulatory Setting. Curr Pain Headache Rep 2016;20:18.

20. Kharouba J, Ratson T, Somri M, Blumer S. Preemptive Analgesia by Paracetamol, Ibuprofen or Placebo in Pediatric Dental Care: A Randomized Controlled Study. J Clin Pediatr Dent 2019;43:51-55. [CrossRef]

21. Munro A, Sjaus A, George RB. Anesthesia and analgesia for gynecological surgery. Curr Opin Anaesthesiol 2018;31:274-9.

22. Ridderikhof $M L$, Lirk $P$, Goddijn $H$, Vandewalle $E$, Schinkel $E$, Van Dieren S, et al. Acetaminophen or Nonsteroidal Anti-Inflammatory Drugs in Acute Musculoskeletal Trauma: A Multicenter, Double-Blind, Randomized, Clinical Trial. Ann Emerg Med 2018;71:357-368.e8. [CrossRef] 
23. Triantafillidis JK, Merikas E, Nikolakis D, Papalois AE. Sedation in gastrointestinal endoscopy: current issues. World J Gastroenterol 2013;19:463-81. [CrossRef]

24. Triantafillidis JK, Merikas E, Nikolakis D, Papalois AE. Sedation in gastrointestinal endoscopy: current issues. World J Gastroenterol 2013;19:463-81. [CrossRef]

25. ASGE Standards of Practice Committee, Early DS, Lightdale JR,
Vargo JJ 2nd, Acosta RD, Chandrasekhara V, et al. Guidelines for sedation and anesthesia in Gl endoscopy. Gastrointest Endosc 2018;87:327-37. [CrossRef]

26. Orel R, Brecelj J, Dias JA, Romano C, Barros F, Thomson M, et al. Review on sedation for gastrointestinal tract endoscopy in children by non-anesthesiologists. World J Gastrointest Endosc 2015;7:895-911. [CrossRef] 